\title{
Sunward propagating Pc5 waves observed on the post-midnight magnetospheric flank
}

\author{
P. T. I. Eriksson ${ }^{1}$, L. G. Blomberg ${ }^{1}$, S. Schaefer ${ }^{2}$, and K.-H. Glassmeier ${ }^{2}$ \\ ${ }^{1}$ Space and Plasma Physics, School of Electrical Engineering, Royal Institute of Technology, Stockholm, Sweden \\ ${ }^{2}$ Institute for Geophysics and Extraterrestrial Physics, Technical University of Braunschweig, Braunschweig, Germany \\ Received: 3 May 2007 - Revised: 22 February 2008 - Accepted: 8 April 2008 - Published: 11 June 2008
}

\begin{abstract}
The overall focus on the driver of toroidal Pc5 waves has been on processes located at or acting on the dayside magnetopause and dayside flanks of the magnetosphere. These processes can generate waves that propagate tailward in the magnetosphere. However, an increasing number of studies, both theoretical and experimental, have looked at waves propagating sunward and that are caused by processes in the magnetotail. Here we present an ultra low frequency (ULF) wave observed in the post-midnight/morning sector of the magnetosphere at $L=16 R_{E}$. The wave has a toroidal mode polarization. We estimate the azimuthal wave number to $m=3$, consistent with a toroidal mode type pulsation. The positive sign indicates that the wave is propagating sunward and this is confirmed by looking at the Poynting flux of the wave. The frequency of the wave is not constant with time but shows a small increase in the beginning of the event up to over $2.0 \mathrm{mHz}$. Then the frequency decreases to $1.0 \mathrm{mHz}$. This decrease coincides with a drop in the total magnetic field strength and we speculate if this is related to an observed reversal of the sign of the interplanetary magnetic field (IMF) By-component. This event occurs during relatively quiet magnetospheric conditions with a solar wind speed of approximately $400 \mathrm{~km} / \mathrm{s}$. Thus this event is highly likely to be driven by a source in the magnetotail and the change in frequency is an excellent example that the frequency of an ULF wave may be modulated by changes of the plasma parameters on the resonant field line.
\end{abstract}

Keywords. Magnetospheric physics (MHD waves and instabilities; Solar wind-magnetosphere interactions)

Correspondence to: P. T. I. Eriksson

(tommy.eriksson@ee.kth.se)

\section{Introduction}

ULF waves are common phenomena in the magnetospheric dawn and dusk sectors. Despite their frequent appearance the source or sources of these waves are still under considerable debate. One of the central concepts in the theory of ULF waves is that of the field line resonance (FLR), which was first discussed by Tamao (1965) but made widely known to the scientific community by the papers of Southwood (1974) and Chen and Hasegawa (1974). An FLR is the coupling of energy from a fast mode wave to an Alfvén wave at a location where the local Alfvén eigenfrequency of the field line matches the frequency of the fast wave. The first candidate suggested as a source for the fast mode waves was the Kelvin-Helmholtz instability acting at the magnetopause. This driver can account for many of the observed features of field line resonance. It cannot, however, explain the multiple frequencies observed simultaneously.

A number of studies have shown that there seems to be a preferred set of frequencies for FLR, often quoted to be 1.3, 1.9, 2.6 and $3.4 \mathrm{mHz}$ (e.g. Samson et al. 1991, Ruohoniemi et al. 1991, Mathie et al. 1999). A theoretical explanation for these frequencies can be found in a series of papers by Kivelson and co-workers (Kivelson and Southwood 1985, 1986). They showed that the magnetosphere could act as a resonant cavity for the fast mode and that the observed frequencies would be the eigenfrequencies of this cavity. Walker et al. (1992) noted that a waveguide is a better description since the magnetosphere is open downtail. This waveguide description has now been elaborated by a number of authors (for a review of the waveguide theory development see Wright and Mann, 2006). The predominant frequencies are not totally undisputed, however. Baker et al. (2003) for instance conducted a large statistical study based on CANOPUS magnetometer data. They confirmed some of the previously observed Pc5 pulsation characteristics like the dawn/dusk occurrence asymmetry and the

Published by Copernicus Publications on behalf of the European Geosciences Union. 
increase in Pc5 band power with increasing solar wind speed. They did not find any preferred frequencies however. Mazur and Leonovich (2006) on the other hand gave an explanation for the stability of the observed frequencies. They used a parabolic geometry for the magnetosphere and an Alfvén velocity distribution based on satellite data and showed that the eigenfrequencies of their system closely matched the observed frequencies. One of their suggestions was that for an eigenmode of the system to develop its characteristic parameters have to remain unchanged for several periods. Thus geomagnetically quiet conditions are required for these modes to develop.

There are two fundamentally different ways to construct a waveguide description of the magnetosphere. One is where the magnetospheric flanks are considered and are modeled using a box-geometry where the coordinates usually are chosen to be $x$ radially outward from the Earth, $y$ along the flank positive tailward and $z$ is in the direction of the magnetic field (where the field lines are considered straight) (e.g. Wright, 1994; Rickard and Wright, 1994, 1995; Wright and Rickard, 1995).

The other type of magnetospheric waveguide is the magnetotail. This second type of waveguide is inherently different from the first type as the ambient magnetic field is directed along the waveguide as opposed to across it. Allan and Wright (1998) used the following box model for the magnetotail waveguide. The coordinate $x$ extends from a position in the tail at $x=0$ to $x=x_{E}$, the "Earth". The magnetic field is assumed to be uniform in the $\mathrm{x}$-direction, $\mathbf{B}=B_{0} \hat{\mathbf{x}}$. The $z$-coordinate extends across the waveguide so the southern magnetopause is assumed to be located at $z=-z_{M}$ and the northern magnetopause located at $z=z_{M}$. The y-coordinate can be regarded as the azimuthal component. In this geometry, field line resonance of the flank type is not possible. The magnetotail waveguide shows dispersion so that waves with the largest $k_{\| \mid}$and smallest $k_{z}$ propagate fastest down the waveguide, while components with smaller $k_{\|}$and larger $k_{z}$ propagate much slower down the waveguide, similar to the dispersion in the flank waveguide.

Mathews et al. (2004) presented multi-instrument observations of periodic, multiple east-west aligned auroral arcs in the dusk sector that were propagating sunward and equatorward from the poleward boundary of the auroral oval. They showed that the periodicity of the arcs matched the periodicity of fluctuations of the local magnetic field. They furthermore suggested that the observed sunward propagation of the arcs can be explained if they are excited by field line resonances that are driven by waveguide mode waves that propagate sunward from the magnetotail. In a recent study by Takada et al. (2006), ULF waves in the magnetotail lobes were investigated using the Cluster spacecraft. This study focused on the transport of energy from the magnetotail towards the Earth by these waves. Their events were in general not associated with high solar wind speeds and they suggested that these waves were caused by an internal source in the plasmasheet or plasmasheet boundary layer. Also Zheng et al. (2006) used Cluster to study ULF waves in the magnetotail. The waves were observed to propagate westward (anti-sunward for this event), to have amplitude and phase structure of an FLR as deduced from ground-based magnetometers and to have a frequency of $1.1 \mathrm{mHz}$. This event occurred during high solar wind speed conditions (average solar wind speed of $680 \mathrm{~km} / \mathrm{s}$ ), suggesting a Kelvin Helmholtz instability at the magnetopause as the driving mechanism although the authors could not rule out other possibilities.

This paper focuses on a Pc5 pulsation in the post-midnight magnetotail flank observed by the Cluster spacecraft. The wave shows two properties that deserve attention. Firstly the wave appears to propagate sunward. Secondly the frequency is changing with time. The paper is organised as follows. Section 2 describes the instruments used in this study. Section 3 presents our observations and Sect. 4 discusses our observations with previously published results. Finally Sect. 5 gives a short summary of our observations.

\section{Instrumentation}

This study mainly focuses on measurements from the Cluster spacecraft. We use data from the electric field and waves instrument (EFW, Gustafsson et al., 1997) and the fluxgate magnetometer instrument (FGM, Balogh et al., 1997). Figure 1 shows an illustration of the Cluster orbits (red lines) plotted from 22:00 UT 21 July 2004 to 01:00 UT 22 July 2004. The magnetic field lines on which the spacecraft are located at 00:00 are plotted and also the outermost field lines of the magnetosphere. The magnetic field is calculated using the T01 model (Tsyganenko, 2002a, b). Figure 1a shows the projection in the GSM xy-plane, Fig. 1b shows the projection in the xz-plane and Fig. 1c shows the magnetic footprint of s/c 3 (red circles). The locations of the IMAGE magnetometers and four Russian magnetometers (black circles) are also shown. The footprint of Cluster moves westward during the time interval so that at 01:00 the footpoint is located at the southern tip of Svalbard. At 23:34 UT when the oscillations appear in the electric field, Cluster is located at [ -9.25 , $-12.0,4.00] R_{E}$ GSE-coordinates and when the oscillations no longer are present at around 01:15 UT, Cluster is located at $[-10.3,-12.5,3.05] R_{E}$. Thus the satellites are moving tailward and towards the magnetic equator during this event. To get a better understanding of how the satellites are positioned we have plotted their location with respect to $L$-value, angle from the magnetic equator and magnetic local time (MLT) in Fig. 1d. These values have been calculated from just the IGRF model of the magnetic field and the magnetic equator is defined as the plane with $z=0$ in SM-coordinates so that the absolute values may differ greatly from the real ones (apart from MLT) but the relative values are still meaningful. For this event the spacecraft separation is of the order of $1000 \mathrm{~km}$. 
(a)

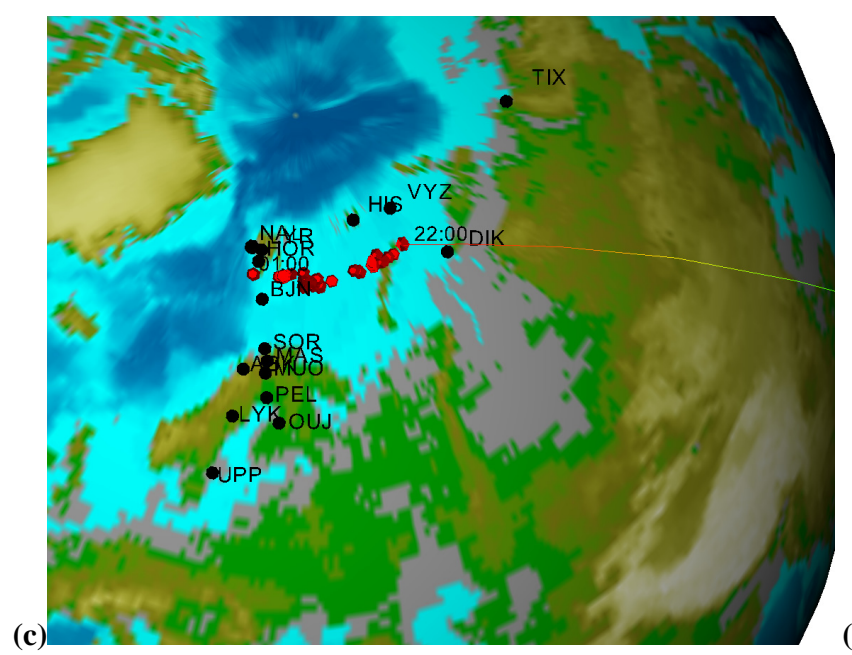

(b)
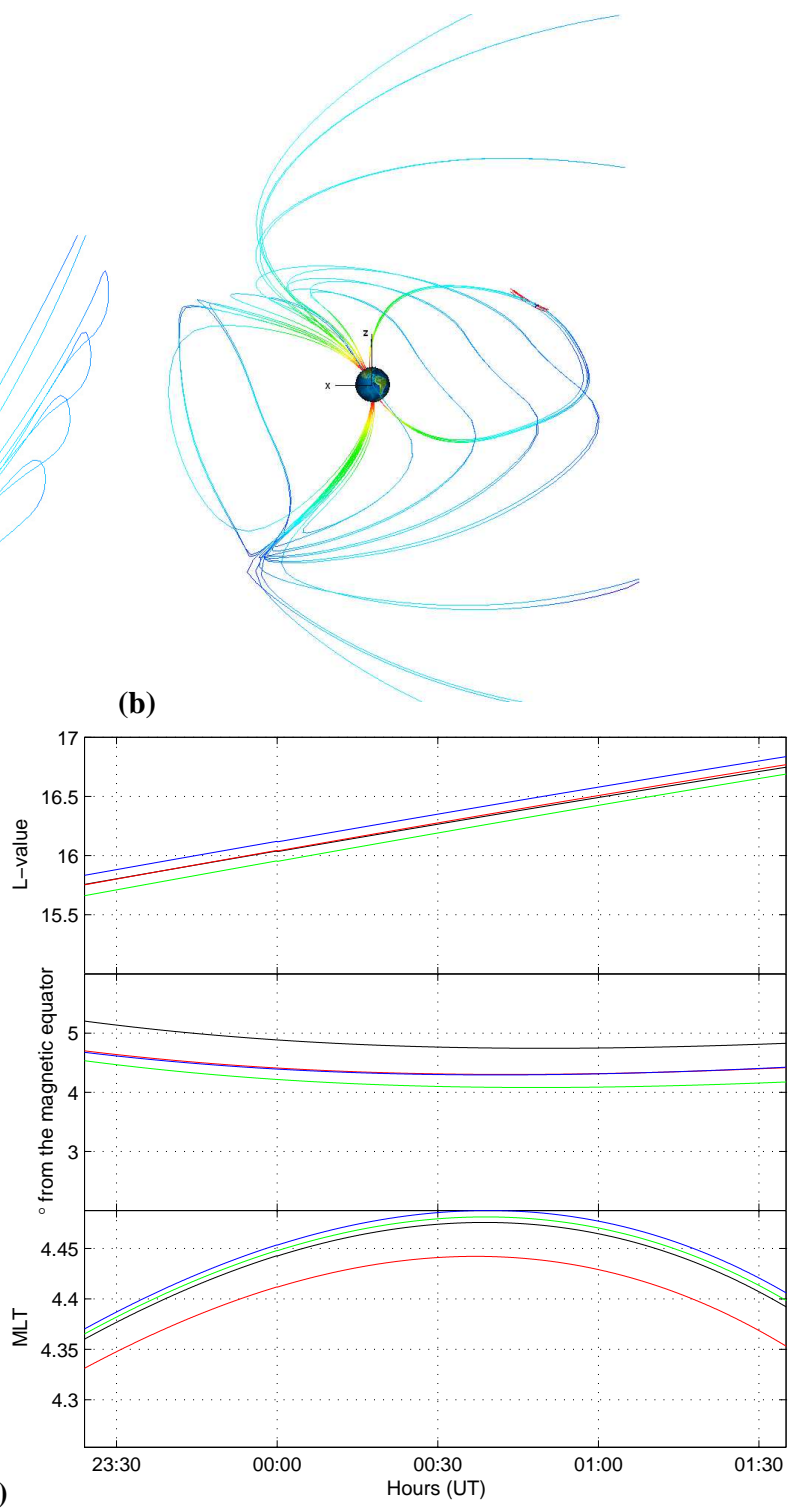

(d)

Fig. 1. Sketch of the magnetosphere at 00:00 UT from the T01 model, the field lines which the Cluster satellites are located and the Cluster orbits (red lines) in GSM coordinates. (a) Projection in the GSM xy-plane. (b) Projection in the xz-plane. (c) Magnetic footprint for s/c 3 (red circles). Ground-based magnetometers are indicated by black circles. (d) Clusters position with respect to L-value (calculated from the IGFR model), angle from the dipole magnetic equator and magnetic local time (MLT).

We also include solar wind observations from the ACE spacecraft. At 22:00 UT 21 July 2004 ACE was located at [234, $-40.2,22.7] R_{E}$ (GSE-coordinates). From the average solar wind speed during the interval we estimate the propagation time from ACE to the Earth to $63 \mathrm{~min}$. Figure 2 shows field and particle measurements from ACE from 22:00 UT to 01:00 UT. The solar wind speed is approximately $400 \mathrm{~km} / \mathrm{s}$ and the density approximately $2.5 \mathrm{~cm}^{-3}$ during the interval. The most prominent feature is a reversal in the IMF $B_{y}$ component at 23:35 UT. This reversal is associated with a drop in the geomagnetic magnetic field strength at the loca- tion of Cluster and we shall later discuss the implications of it.

\section{Observations}

In Fig. 3 we have plotted the duskward electric field measured by all four Cluster spacecraft. The oscillations are clearly visible as they are superimposed on a quiet background. The signal is strikingly similar on all four satellites which gives an indication of the scale-size of the wave. Also 

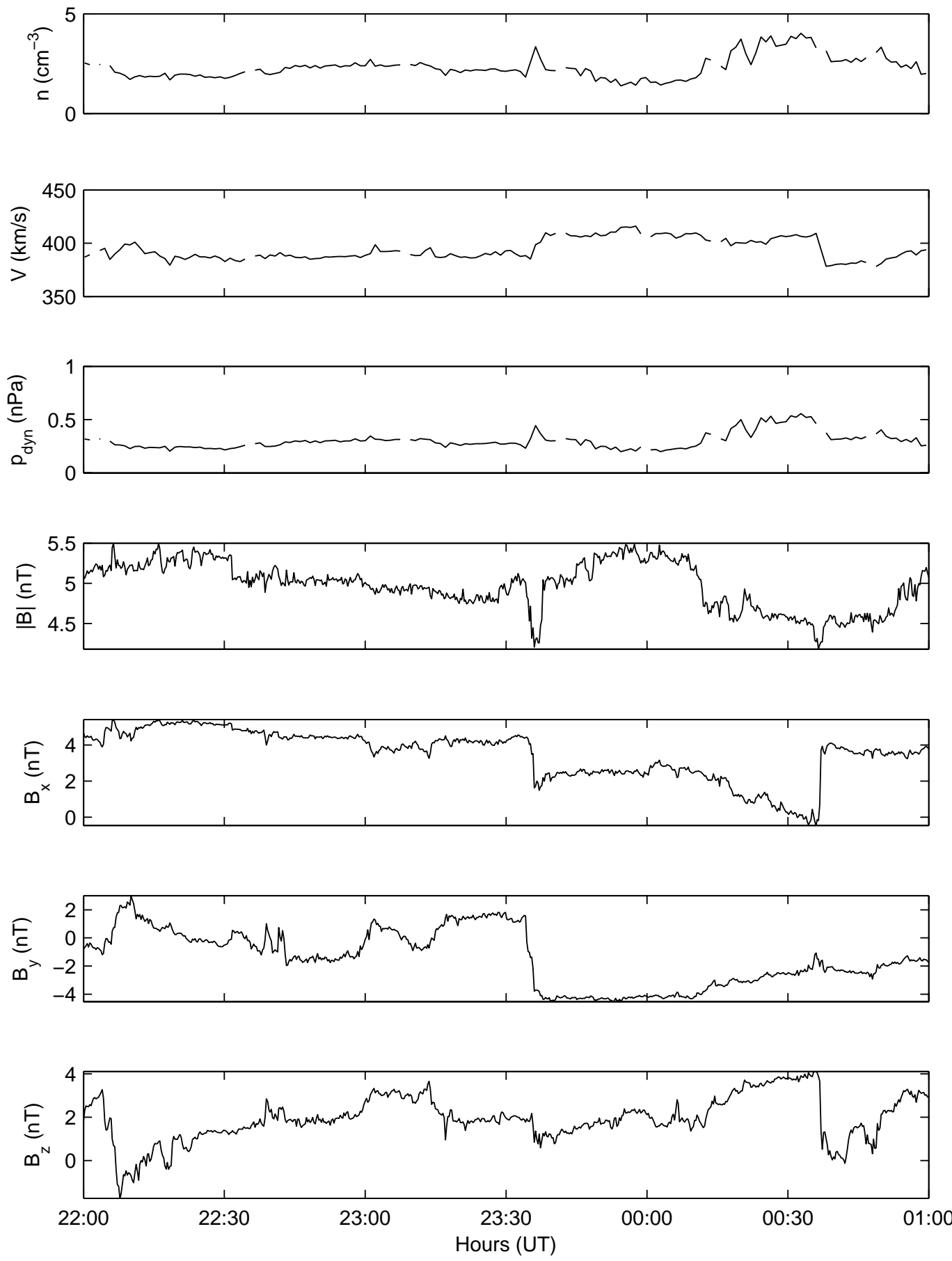

Fig. 2. Solar wind parameters measured by the ACE spacecraft.

visible to the naked eye is the changing distance in time between the peaks of the wave indicating that the frequency is changing with time. In particular, there is an almost linear change in the electric field strength from 00:26 UT to 00:33 UT, most prominent on $\mathrm{s} / \mathrm{c} 1$. We will return to this in the discussion section.
To obtain the polarisation of the wave we have transformed the electric and magnetic field into a field-aligned coordinate system. The coordinate system is the same as the one used by Eriksson et al. (2005). Before performing the transformation we calculate the third component of the electric field by assuming that the electric field is perpendicular to the magnetic field (i.e. $\boldsymbol{E} \times \boldsymbol{B}=0$ ). Figure 4 shows the electric 


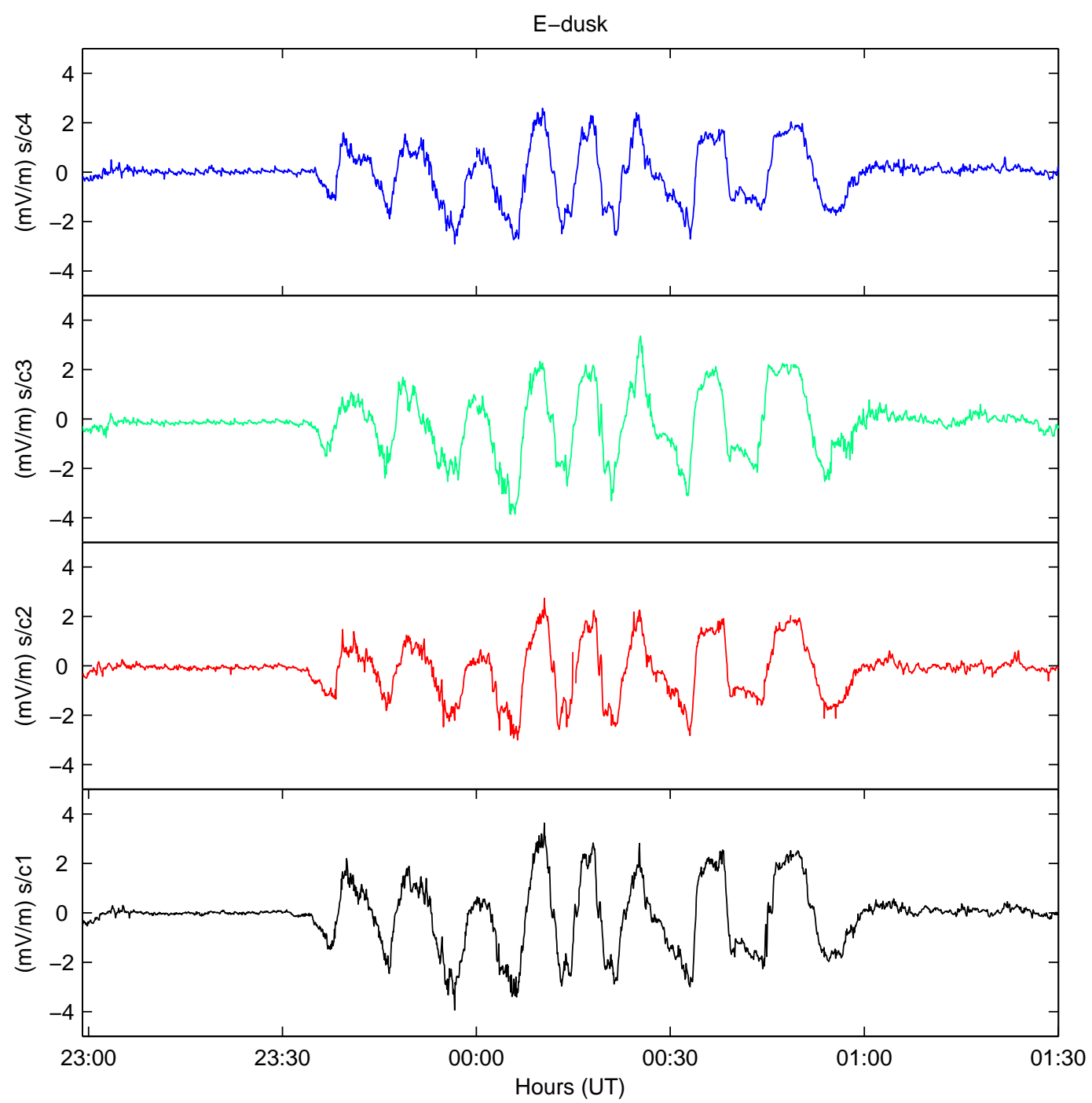

Fig. 3. Duskward electric field measured by s/c 1 (black), s/c 2(red) s/c 3 (green), s/c 4 (blue).

and magnetic field measured by s/c 4 in field-aligned coordinates. The data have been bandpass filtered with a fifth order Butterworth filter with passband $0.9-4 \mathrm{mHz}$. Inspection of the two electric field components reveals that the radial component is the dominant, with a peak amplitude of approximately $6 \mathrm{mV} / \mathrm{m}$ which is three times that of the eastward electric field component which has a peak amplitude of $2 \mathrm{mV} / \mathrm{m}$. The dominant component of the magnetic field is the eastward component, consistent with what is seen in the electric field, although the amplitude of the magnetic field component seems more strongly modulated than the amplitude of the electric field component.

An important property of Pc5 pulsations is the azimuthal wave number. From the observed toroidal polarisation of this wave we expect it to have a small azimuthal wave number. As discussed by Eriksson et al. (2006), there are primarily two methods for determining the azimuthal wave number. One is to compute the analytic signal for the wave. The imaginary part of the analytic signal is the instantaneous phase and thus the phase difference between two spacecraft can be obtained. The other is to compute the cross spectral density between the signals from two spacecraft. Again the phase (difference) can be extracted by looking at the imaginary part of the cross spectral density. The second method is more suitable for waves with small $m$ and thus this is the one we will use here as we shall see that this event has a small azimuthal wave number. By calculating the phase difference between the six different satellite pairs we obtain six different estimates of $m$, all of which gives $m=3$ with little deviation. Obviously we need to consider that the frequency is not constant during this event and the question then arises at what frequency the phase difference should be taken. Fortunately the cross spectral density yields a high coherency (0.99) at $1.9 \mathrm{mHz}$. So we use the phase difference at this frequency. 
Field in field-aligned coordinates for s/c 4

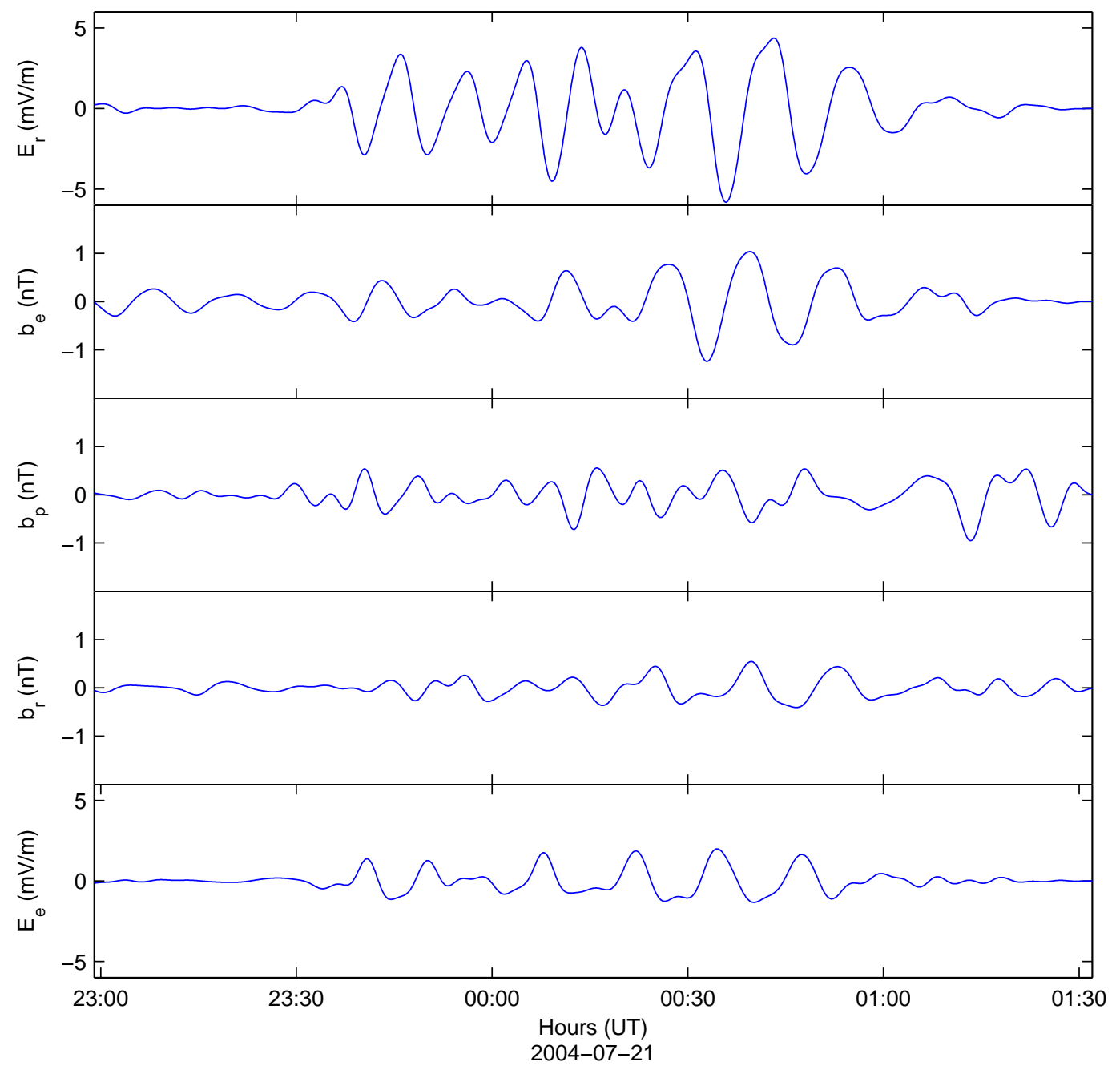

Fig. 4. Field in field-aligned coordinates for s/c 4 .

To study the change in frequency in more detail we have calculated a spectrogram from the duskward electric field component measured by s/c 1 . The spectrogram is calculated as follows. The electric field data are divided into segments, each with the length $2048 \mathrm{~s}$ and with an overlap of 1900 s. A Hamming window of the same size is then applied to each segment. The number of frequency points used to calculate the discrete Fourier transforms is 1024. The result is plotted in Fig. 5 with time on the x-axis, frequency on the y-axis and the logarithm of the intensity is given by a colour-scale. At the beginning of the event the wave frequency is approximately $1.5 \mathrm{mHz}$ and it increases gradually to more than $2 \mathrm{mHz}$ at 00:15 UT. From Fig. 3 we saw that the duskward electric field had an almost linear behaviour between 00:26 UT and 00:33 UT. This is reflected in the spectrogram as a rapid decrease in the frequency. After approximately 00:33 UT the wave displays a slower decrease in frequency to almost $1.0 \mathrm{mHz}$ at the end of the event at around
01:00 UT. Of course it is questionable to speak of the frequency as something instantaneous but this analysis at least highlights the changing nature of the wave.

The changing frequency of this event naturally raises the question of the underlying cause. The travel time approximation (e.g. Schulz, 1996) gives $\omega \sim\left(\int \frac{d s}{v_{A}}\right)^{-1}$ for the eigenfrequency of a magnetic field line, where $v_{A}$ is the Alfvén speed and the integrations is performed along the entire field line. Thus an increase in the magnetic field strength causes an increase in the eigenfrequency and an increase in the plasma density causes a decrease in the eigenfrequency. In Fig. 6 we have plotted the absolute magnetic field strength measured by s/c 3 (green curve) and the field strength at the location of s/c 3 computed from the T01 model (pink curve). The behaviour of the magnetic field at the location of the other three satellites closely resembles that of s/c 3 so we have omitted those for clarity. At the start of the event at 23:34 UT the field strength is approximately $13.2 \mathrm{nT}$ followed by a small 


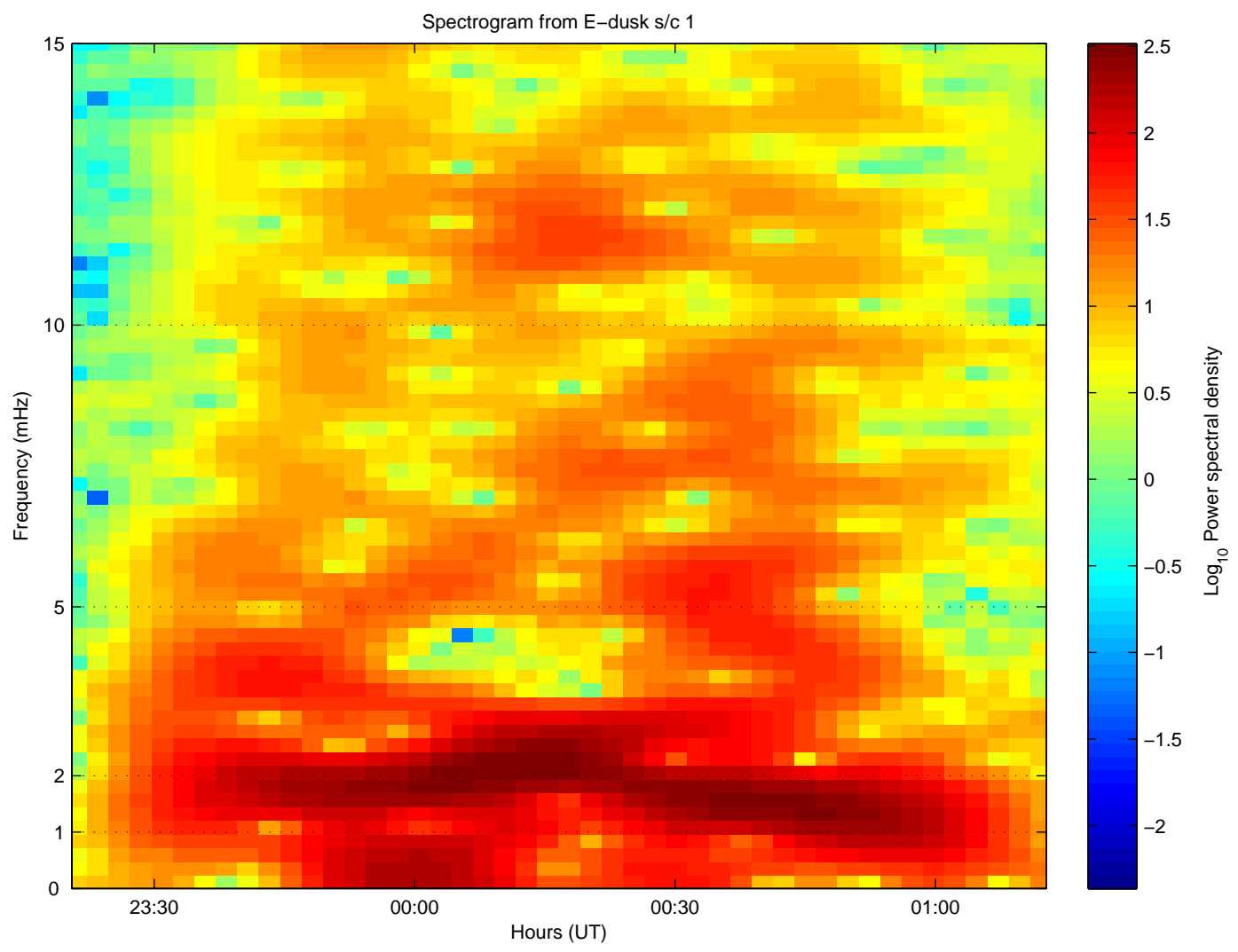

Fig. 5. Spectrogram calculated from the duskward electric field component from s/c 1 .

depression at around 23:37 UT and a spike at 23:40 UT. The spike is followed by another depression reaching a minimum of 12.2 nT at 23:45 UT. From 23:53 UT the field strength increases from $12.2 \mathrm{nT}$ up to $13.9 \mathrm{nT}$ at 00:02 UT after which the field seems to oscillate around $13.5 \mathrm{nT}$ until 00:33 UT. From 00:33 UT to 00:40 UT there is a drop in the magnetic field strength down to $10.7 \mathrm{nT}$ after which the field continues to decrease down to $8.3 \mathrm{nT}$ at 01:13 UT apart from a small peak at 00:48 UT. The magnetic field strength continues to decrease and reaches a minimum of $8.6 \mathrm{nT}$ at 01:13 UT. As for the model field, it shows in overall an overestimate of the field strength but the qualitative behaviour is similar. This makes us confident that the overall trend of the measured magnetic field is due to global variations of the geomagnetic field rather than being a localised behaviour and accordingly these changes would affect the eigenfrequency of the field lines. The model field decreases from the beginning of the plot to $13.9 \mathrm{nT}$ at 23:30 UT. It then stays fairly constant above $14 \mathrm{nT}$ until 00:15 UT where it starts to drop to $12.3 \mathrm{nT}$ at 00:20 UT. From 00:40 UT it makes another drop down to $10.5 \mathrm{nT}$ at 00:55 UT. At 00:10 the model field start to recover again increasing to $13.6 \mathrm{nT}$ at $01: 35 \mathrm{UT}$, mimicking the behaviour of the real magnetic field although the amplitude is overestimated. Our interpretation of the large scale behaviour of the magnetic field is illustrated by the dash-dotted line. Here we have assumed that the smaller wiggles in the magnetic field are small scale (local) variations at the position of the spacecraft and that it is the behaviour marked by the dash-dotted line that is important for the observed change in frequency.

The sign of the azimuthal wave number is important here because it indicates the direction of propagation of the wave. To confirm the direction of propagation we turn our attention to the Poynting flux. In Fig. 7 we have plotted the instantaneous Poynting flux in field-aligned coordinates calculated from $\mathrm{s} / \mathrm{c} 1$ using the same bandpass filter as previously. The red curve is the radial component (positive outward, away from the Earth), green is the eastward component and blue is the field-aligned component. If we first look at the eastward component we see that it is predominantly positive for the entire event. The radial component is also positive for most of the interval with some exceptions in the middle of the interval. Energy is thus transported away from the midnight magnetotail towards the dayside. We note that the parallel component of the Poynting flux oscillates around zero during the majority of the time interval except for the very beginning. The first period of oscillations in the parallel component of the Poynting flux (corresponding to half a period of the wave) has a positive offset from zero so that for this time period energy is transported in the direction of the magnetic 


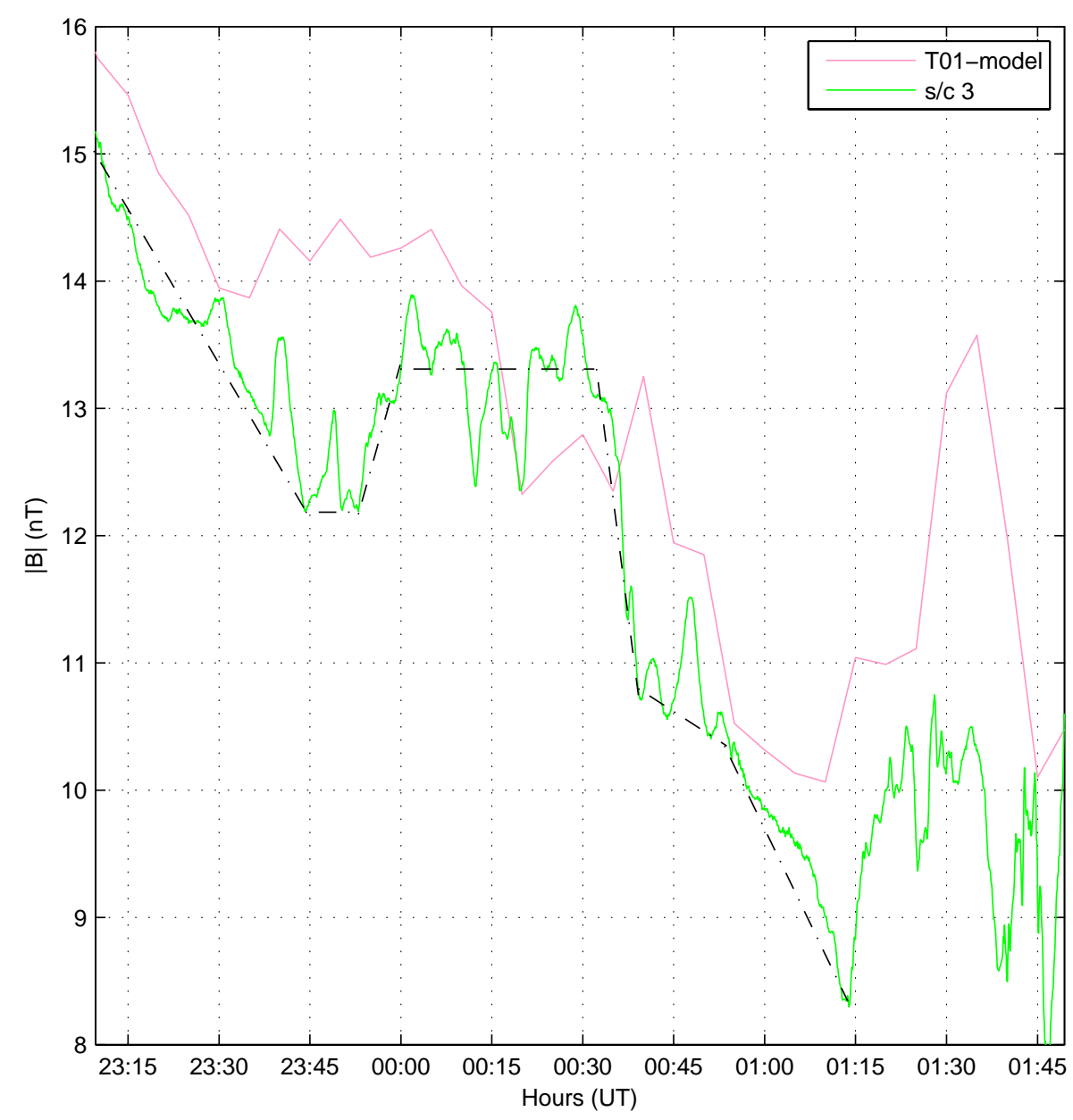

Fig. 6. Absolute magnetic field strength measured by s/c 3 (green curve) and from the T01 model at the location of s/c 3 . The dash-dotted line is our interpretation of the large scale behaviour of the magnetic field strength.

field. This is followed by a negative value of the Poynting flux, suggesting that the wave has been reflected from the northern ionosphere. After 00:00 UT the oscillations in the parallel component of the Poynting flux are approximately centred around zero, indicative of a standing wave along the geomagnetic field.

In Fig. 8a we have plotted the duskward electric field from all four satellites in the interval where the oscillations appear in the Cluster data. We can see that the wave is first observed by s/c 2 (red) at approximately 23:34 UT followed by s/c 3 (green), s/c 1 (black) although the difference between s/c 3 and s/c 1 is subtle and last by s/c 4 at 23:35 UT. Returning to Fig. 1d the third panel shows the magnetic local time of the satellites with s/c 2 the spacecraft closest to midnight. Then there is a gap to the three other satellites which are closely spaced together with s/c $1, \mathrm{~s} / \mathrm{c} 3$ and s/c 4 in increasing order. This separation of the satellites can explain the arrival order of the wave although s/c 3 sees the wave before s/c 1 but $\mathrm{s} / \mathrm{c} 1$ is closer to midnight than s/c 3 . This discrepancy can be explained if we consider that the alignment of the wave phase front does not need to be in the east-west sense defined by magnetic local time. However for the spacecraft pair 2 and 4 that have a fairly large separation in azimuth (MLT) the difference in arrival time becomes quite clear.

As for the disappearance of the wave, we have plotted the end of the wave event in Fig. 8b. Again we have looked at the duskward electric field from all four spacecraft. Unlike the start of the event all of the curves follow each other quite closely marking a temporal change in the evolution of the wave. The damping is clearly evident with a peak electric field of approximately $2 \mathrm{mV} / \mathrm{m}$ at 00:47 UT and a full wave cycle later at 01:02 UT the electric field is only $0.25 \mathrm{mV} / \mathrm{m}$. This means that the wave electric field has been reduced by a factor of 8 during $15 \mathrm{~min}$. After 01:10 UT the wave is no longer visible in the electric field data with the exception of s/c 3 that sees the wave for approximately another half of a wave period. By allowing a complex wave frequency, $\omega \rightarrow \omega+i \gamma$ we can calculate the damping coefficient $\gamma$. If we assume the wave starts to damp at 00:47 UT, which coincided well with the drop in the total magnetic field 


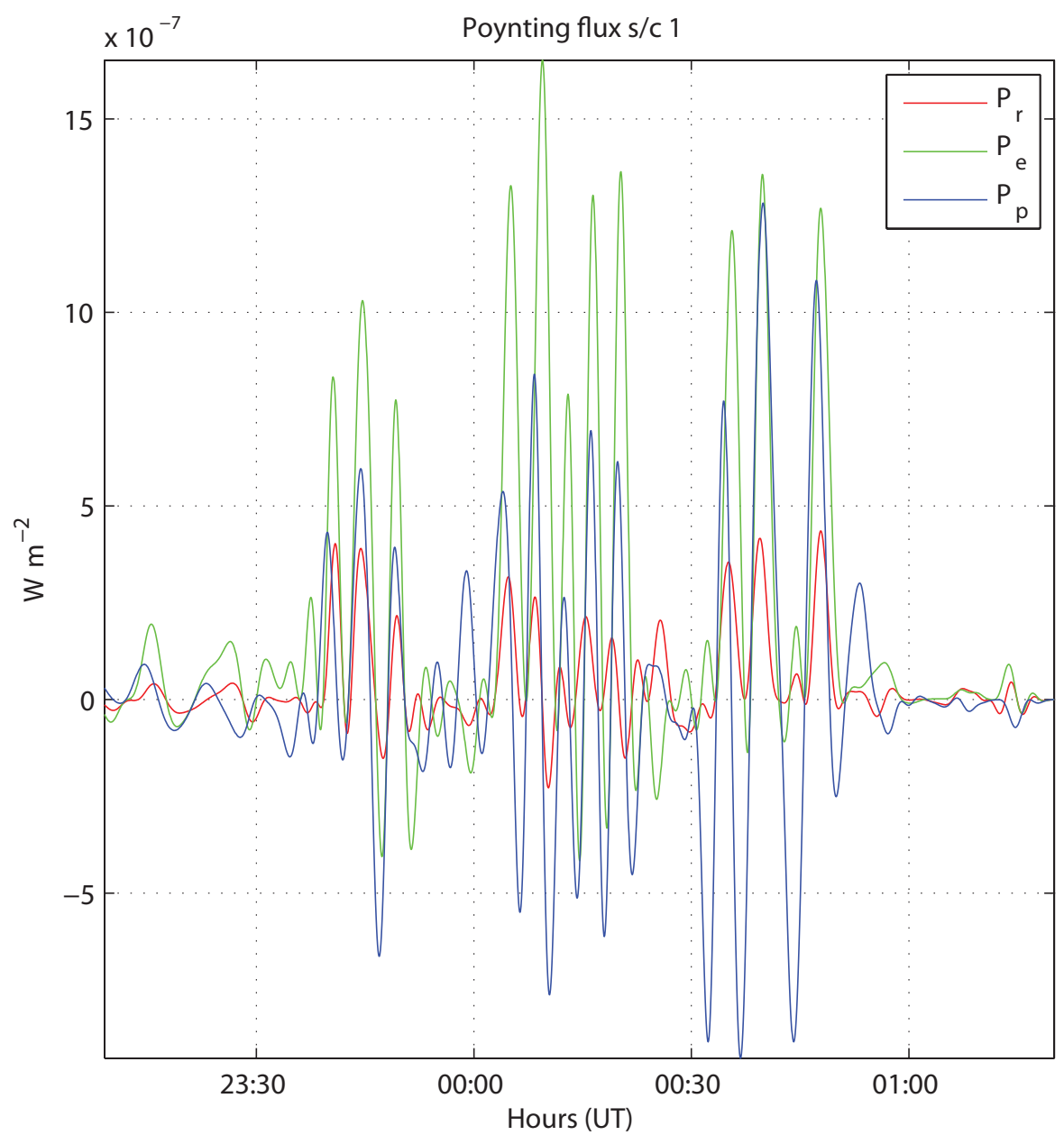

Fig. 7. Poynting flux in field-aligned coordinates calculated from s/c 1 . Blue line is the field-aligned component, green the eastward component and red the radial component.

strength, the damping should be described as $e^{-\gamma \Delta t}=1 / 8$, with $\Delta t=15 \mathrm{~min}$. From this we obtain $\gamma=2.3 \times 10^{-3} / \mathrm{s}$. This is a large number for $\gamma$, which is normally considered to be only a fraction of the wave frequency $(\omega)$ (e.g. Newton et al., 1978). The value is not unrealistic however, Glassmeier et al. (1984) reported values of approximately $6 \times 10^{-3} \mathrm{~s}^{-1}$ for $\gamma$.

\section{Discussion}

The relatively small amplitude observed for the magnetic field is consistent with the findings of Chen and Kivelson (1991). They studied ULF waves in the lobes $\left(-10 \lesssim X_{\mathrm{GSM}} \lesssim-23 R_{E}\right)$ using data from the ISEE 2 satellite and found the magnetic field amplitude to be below $0.8 \mathrm{nT}$ $90 \%$ of the time for transverse waves in the frequency range 1 to $5 \mathrm{mHz}$.

For the discussion of the changing frequency we need to establish what parameters control the eigenfrequency of a ge- omagnetic field line. The problem of calculating the eigenfrequencies for a closed geomagnetic field line has attracted the interest of many authors. As mentioned earlier, in the WKB approximation the (toroidal) eigenfrequency is dependent of the magnetic field strength, the plasma density and the field line length. For the poloidal mode it can be shown (e.g. Klimushkin, 1998a, b) that the eigenfrequency is also affected by field line curvature. In the real case the eigenfrequency of the toroidal mode is also affected by field line curvature (D. Klimushkin, private communication). More sophisticated models have also been developed although these models have to be solved numerically in general. Rankin et al. (2000) numerically solved the MHD equation for a cold plasma with the Tsyganenko 96 model as the background magnetic field. They showed that the eigenfrequencies of the magnetic field lines in the midnight sector are significantly lower than the corresponding dipole values. Going back to the WKB approximation for the eigenfrequency in a dipole geometry it can be shown that it depends linearly on 

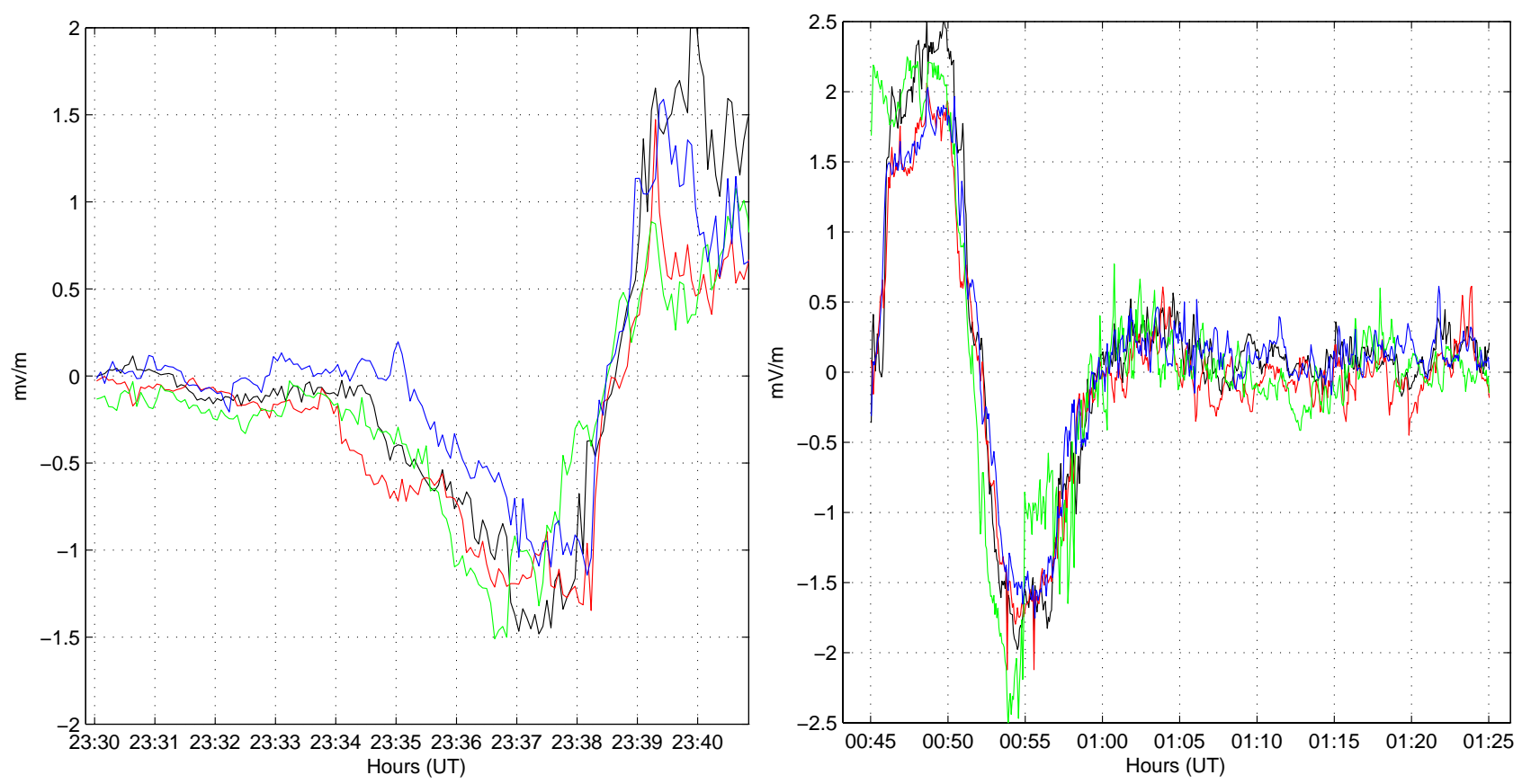

Fig. 8. (a) Duskward electric field measured by s/c 1 (black), s/c 2(red) s/c 3 (green), s/c 4 (blue) for the beginning of the event. (b) Same as in (a) but for the end of the event.

the equatorial Alfvén speed and accordingly it depends linearly on the equatorial magnetic field strength. As a zeroth order approximation we can naively assume that the local measurement of the field strength by Cluster is representative of the entire field line. From the measurements of the field strength we saw that it increased from $12.2 \mathrm{nT}$ at 23:53 UT to $13.9 \mathrm{nT}$ at 00:02 UT., i.e. approximately a $14 \%$ increase. The frequency shows approximately a 30\% increase from 23:45 UT to 00:15 UT, with the major part of the change occurring after 00:00 UT. So the frequency increases more than predicted by the WKB theory but the causal relationship holds with the magnetic field strength increasing before the frequency. From 00:33 UT to 00:40 UT the magnetic field dropped approximately $20 \%$. The frequency starts to drop fairly rapidly at approximately 00:26 UT until approximately 00:30 UT, consistent with the non-sinusoidal behaviour of the electric field. Here the causal relationship appears to be violated and we can only speculate that it is due to the imperfectness of a point measurement being representative of the field strength along the entire field line. The trend is the same however, since it would have been harder to explain the drop in frequency if the field strength had increased. Also the discrepancy in timing is on the order of five minutes, less then the eigenperiod of the oscillations. After 00:30 UT the frequency continues to decrease but more slowly. The drop in the magnetic field strength observed at 00:33 UT corresponds well to the reversal in the IMF $B_{y}$-component if we assume that the travel time from ACE to the Earth is given by the solar wind speed, i.e. the magnetic field structure is frozen into the solar wind plasma. If we assume that the drop in the magnetic field strength is caused by the reversal in the IMF $B_{y}$-component this would also shed some light on the discrepancy in timing. The disturbance in the magnetic field caused by the IMF reversal would propagate tailward affecting different parts of the geomagnetic field line at different times and as we can see from Fig. 1, Cluster is located almost at the most "distant part" of the field line.

To see how the plasma density is behaving we have looked at the probe to spacecraft potential as measured by the EFW instrument. The relationship between the probe potential and the electron density has been studied several authors (e.g. Pedersen et al., 2001, for Cluster, Ishisaka et al., 2001, for Geotail). For our event, the qualitative behaviour of the probe potential is similar to that of the magnetic field strength, with an increase in the beginning of the event followed by a plateau in the middle and a decrease towards the end of the event. So as the probe potential (density) increases approximately at the same time as the frequency increases and vise versa we do not believe that changes in the plasma density plays any major part in the change in frequency.

On the other hand, it is justified to ask the question if the change in frequency can be related to a change in frequency of the driver. This change may be the effect of frequency dispersion in the magnetotail waveguide as described by Allan and Wright (2000). However we believe this to be unlikely because the frequency changes approximately $50 \%$ 
(from $2 \mathrm{mHz}$ down to $1 \mathrm{mHz}$ ) at the same time as the Poynting flux shows that the wave is a standing wave along the geomagnetic field. Thus if the driver changes frequency the wave would no longer appear to be standing in the Poynting flux plot. Movement of the spacecraft is also a factor to consider. From Fig. 1d we see that Cluster moves less than $1 R_{E}$ in the radial direction (monotonically increasing) which is hard to reconcile with the relatively large and non-linear change in frequency. As for the length of the geomagnetic field line at the location of Cluster we have calculated it to be approximately $42 R_{E}$ from the T01-model, deviating no more than $5 \%$ from this value during the time the waves is observed. This suggests that variation of the field line length alone cannot account for the observed frequency change but it may be a smaller piece in a larger puzzle. Also, from just a theoretical point of view, to incorporate the change in frequency from a change in the field line length would require an enormous stretching of the field. Our interpretation of the change in frequency is thus that the parameters that control the field line, in particular the magnetic field, change during the time that the field line reverberates and so forcing the system to adjust to these changes, much like if you pluck a guitar string and then change the tension of the string causing it to change its tone. The rate of occurrence for changes in the frequency of the type studied here is of course yet to be determined. This is also true for changes in frequency from other causes at least to the best of our knowledge. Since reversals in one or several of the components of the interplanetary magnetic field are fairly common and that there almost always is ULF wave activity going on in the magnetosphere suggests that the process studied here has some significance.

The temporal damping observed at the end of the wave event cannot be explained by the cold MHD theory in a slab geometry as the dispersion relation for the shear Alfvén wave reads $\omega^{2}=V_{A}^{2} k_{z}^{2}$ and thus $\omega^{2}$ cannot become negative. However, collisionless damping is non-zero in a curved magnetic field geometry (Newton et al., 1978) which may explain the observed damping. Another explanation for the wave to disappear within a wave period is that the field line has been opened by some reconnection process and thus energy would simply follow the field line anti-sunward. We believe the latter to be unlikely though, as Cluster appears to be well within the region of closed field lines as indicated by the Tsyganenko model. Ionospheric damping is another possible damping mechanism.

From the azimuthal wave number we can estimate the azimuthal wave length and the azimuthal phase speed of the wave. The azimuthal phase velocity can be written as (e.g. Mathie and Mann, 2000), $V_{p}=\left|2 \pi L R_{E} f / m\right|$, where $R_{E}$ is the radius of the Earth and $f$ is the wave frequency and $L$ is the McIlwain parameter. Although this formula is for a dipole geometry it should at least give us the order of magnitude of the phase speed. Taking $f=1.5 \mathrm{mHz}$ as the (average) frequency of the wave we get $V_{p}=320 \mathrm{~km} / \mathrm{s}$. From the difference in arrival time of the wave between the satellites we may also estimate the group velocity of the wave. Again we look for an order of magnitude estimate. Taking the separation between s/c 2 and s/c 4 to be $1000 \mathrm{~km}$ and the difference in arrival time to be 1 min we get for the group velocity $v_{g}=17 \mathrm{~km} / \mathrm{s}$, which is an order of magnitude smaller than the phase velocity. This is in agreement with the results of Rickard and Wright (1995), who showed that the group velocity of a waveguide mode is an order of magnitude smaller than the phase velocity (their Figs. 4 and 5). The group velocity is an order of magnitude larger than the satellite velocity, justifying the latter to be neglected in the calculations above.

As for the sunward propagation of the wave, this is established by the sign of the azimuthal wave number (positive in this case), the average direction of the Poynting flux and by the onset timing on the four spacecraft. This places the source of the pulsations tailward of the location of Cluster. A magnetotail source of Pc5 pulsations has been suggested theoretically by Allan and Wright (2000) and experimentally by Mathews et al. (2004). These tail-waveguide eigenmodes are thought to be generated by Earthward flows of the magnetotail plasma. An example of such an Earthward flow was studied by Volwerk et al. (2005). They observed Earthward plasma flow with the Cluster satellites on 14 August 2004, 23 days after our observations. At the time of their event Cluster was located at $x_{\mathrm{GSE}} \approx-14 R_{E}$, whereas for our event we have $x_{\mathrm{GSE}} \approx-10 R_{E}$. The plasma flow velocity was found to be $v_{x} \approx 600 \mathrm{~km} / \mathrm{s}$ and associated with a decrease in the $\mathrm{x}$ component of the magnetic field and an increase in the $\mathrm{z}$ component, indicating a dipolarisation of the magnetic field. Volwerk et al. also observed ULF waves with a period of approximately $5 \mathrm{~min}$ during the passage of the magnetic structure. The waves propagated tailward but were also observed by ground magnetometers. The findings of Volwerk et al. show that processes can occur which can potentially generate ULF waves not far from the position of our observations. Since direct measurement of a driver of FLRs has proven difficult (e.g. Waters et al., 2002) it is important to get as much indirect information from the observed wave as possible.

\section{Summary and conclusions}

We have reported a Cluster observation of a ULF wave in the post-midnight magnetosphere flank (Cluster approximately located at $[-10,-12,4] R_{E}$ GSE-coordinates). The wave displays two peculiar properties that we have studied in detail. The first is a sunward propagation as inferred from the sign of the azimuthal wave number, the arrival time between the satellites and the direction of the Poynting flux. This leads us to suggest a source in the magnetotail, possible an Earthward flow of plasma. The second property is a nonlinear change in frequency. We relate this change to changes in the parameters that control the eigenfrequency of a field line, in particular we contribute changes in the magnetic field 
strength to most of the observed change in frequency of the wave.

Acknowledgements. Work at the Royal Institute of Technology was partially supported by the Swedish National Space Board and the Alfvén Laboratory Centre for Space and Fusion Plasma Physics. Work at TU Braunschweig was financially supported by the German Bundesministerium für Wirtschaft und Technologie and the Deutsches Zentrum für Luft- und Raumfahrt under contract 50OC 0104. The work was supported by INTAS grant 05-100008-7978.

Topical Editor I. A. Daglis thanks one anonymous referee for her/his help in evaluating this paper.

\section{References}

Allan, W. and Wright, A. N.: Hydromagnetic wave propagation and coupling in a magnetotail waveguide, J. Geophys. Res., 103, 2359-2368, 1998.

Allan, W. and Wright, A. N.: Magnetotail waveguide: Fast and Alfvén waves in the plasma sheet boundary layer and lobe, J. Geophys. Res., 105(A1), 317-328, 2000.

Baker, G. J., Donovan, E. F., and Jackel, B. J.: A comprehensive survey of auroral latitude Pc5 pulsation characteristics, J. Geophys. Res., 108(A10), 1384, doi:10.1029/2002JA009801, 2003.

Balogh, A., Dunlop, M. W., Cowley, S. W. H., et al.: The Cluster magnetic field investigation, Space Sci. Rev., 79, 65-92, 1997.

Chen, L. and Hasegawa, A.: A theory of long-period magnetic pulsations, 1, Steady state excitation of field line resonance, J. Geophys. Res., 79, 1024-1032, 1974.

Chen, S.-H. and Kivelson, M. G.: On ultralow frequency waves in the lobes of the Earths magnetotail, J. Geophys. Res., 96(A9), 15 711-15 723, 1991.

Eriksson, P. T. I., Blomberg, L. G., Walker, A. D. M., and Glassmeier, K.-H.: Poloidal ULF oscillations in the dayside magnetosphere: a Cluster study, Ann. Geophys., 23, 2679-2686, 2005, http://www.ann-geophys.net/23/2679/2005/.

Eriksson, P. T. I., Blomberg, L. G., Schaefer, S., and Glassmeier, K.-H.: On the excitation of ULF waves by solar wind pressure enhancements, Ann. Geophys., 24, 3161-3172, 2006, http://www.ann-geophys.net/24/3161/2006/.

Glassmeier, K. H., Volpers, H., and Baumjohann, W.: Ionospheric Joule dissipation as a damping mechanism for high latitude ULF pulsations: Observational evidence, Planet. Space Sci., 32(11), 1463-1466, 1984.

Gustafsson, G., Boström, R., Holback, B., et al.: The electric field and wave experiment for the Cluster mission, Space Sci. Rev., 79, 137-156, 1997.

Ishisaka, K., Okada, T., Tsuruda, K., Hayakawa, H., Mukai, T., and Matsumoto, H.: Relationship between the Geotail spacecraft potential and magnetospheric electron number density including the distant tail regions, J. Geophys. Res., 106, 6309-6319, 2001.

Kivelson, M. G. and Southwood, D. J.: Resonant ULF waves: A new interpretation, Geophys. Res. Lett., 12, 49-51, 1985.

Kivelson, M. G. and Southwood, D. J.: Coupling of global magnetospheric eigenmodes to field line resonances, J. Geophys. Res., 91, 4335-4351, 1986.

Klimushkin, D. Y.: Resonators for hydromagnetic waves in the magnetosphere, J. Geophys. Res., 103, 2369-2376, doi:10.1029/97JA02193, 1998a.
Klimushkin, D. Y.: Theory of azimuthally small-scale hydromagnetic waves in the axisymmetric magnetosphere with finite plasma pressure, Ann. Geophys., 16, 303-321, 1998b, http://www.ann-geophys.net/16/303/1998/.

Mathews, J. T., Mann, I. R., Rae, I. J., and Moen, J.: Multiinstrument observations of ULF wave-driven discrete auroral arcs propagating sunward and equatorward from the poleward boundary of the duskside auroral oval, Phys. Plasmas, 11, 4, doi:10.1063/1.1647137, 2004.

Mathie, R. A., Mann, I. R., Menk, F. W., and Orr, D.: Pc5 ULF pulsations associated with waveguide modes observed with the IMAGE magnetometer array, J. Geophys. Res., 104, 7025-7036, 1999.

Mathie, R. A. and Mann, I. R.: Observations of Pc5 field line resonance azimuthal phase speeds: A diagnostic of their excitation mechanism, J. Geophys. Res., 105, 10 713-10 728, 2000.

Mazur, V. A. and Leonovich, A. S.: ULF hydromagnetic oscillations with the discrete spectrum as eigenmodes of MHDresonator in the near-Earth part of the plasma sheet, Ann. Geophys., 24, 1639-1648, 2006, http://www.ann-geophys.net/24/1639/2006/.

Newton, R. S., Southwood, D. J., and Hughes, W. J.: Damping of geomagnetic pulsations by the ionosphere, Planet. Space Sci., 26, 201-209, 1978.

A. Pedersen, Décréau, P., Escoubet, C.-P., Gustafsson, G., Laakso, H., Lindqvist, P.-A., Lybekk, B., Masson, A., Mozer, F., and Vaivads, A.: Four-point high time resolution information on electron densities by the electric field experiments (EFW) on Cluster, Ann. Geophys., 19, 1483-1489, 2001, http://www.ann-geophys.net/19/1483/2001/.

Rankin, R., Fenrich, F., and Tikhonchuk, V. T.: Shear Alfvén waves on stretched magnetic field lines near midnight in Earths magnetosphere, Geophys. Res. Lett., 27(20), 3265-3268, 2000.

Rickard, G. J. and Wright, A. N.: Alfvén resonance excitation and fast wave propagation in magnetospheric waveguides, J. Geophys. Res., 99, 13 455-13 464, 1994.

Rickard, G. J. and Wright, A. N.: ULF pulsations in a magnetospheric waveguide: Comparison of real and simulated satellite data, J. Geophys. Res., 99, 3531-3537, 1995.

Ruohoniemi, J. M., Greenwald, R. A., Baker, K. B., and Samson, J. C.: HF radar observations of Pc5 field line resonances in the midnight/early morning MLT sector, J. Geophys. Res., 96, $15697-$ 15710, 1991.

Samson, J. C., Greenwald, R. A., Ruohoniemi, J. M., Hughes, T. J., and Wallis, D. D.: Magnetometer and radar observations of magnetohydrodynamic cavity modes in the Earth's magnetosphere, Can. J. Phys., 69, 929-937, 1991.

Schulz, M.: Eigenfrequencies of geomagnetic field lines and implications for plasma-density modeling, J. Geophys. Res., 101(A8), 17 385-17 398, 1996.

Southwood, D. J.: Some features of field line resonances in the magnetosphere, Planet. Space Sci., 22, 483-491, 1974.

Takada, T., Nakamura, R., Baumjohann, W., Seki, K., Vörös, Z., Asano, Y., Volwerk, M., Runov, A., Zhang, T. L., Balogh, A., Paschmann, G., Torbert, R. B., Klecker, B., Rème, H., PuhlQuinn, P., Canu, P., and Décréau, P. M. E.: Alfvén waves in the near-PSBL lobe: Cluster observations, Ann. Geophys., 24, 1001-1013, 2006, http://www.ann-geophys.net/24/1001/2006/. 
Tamao, T.: Transmission and coupling resonance of hydromagnetic disturbaces in the non-uniform Earth's magnetosphere, Science Reports of Tohoku University, Series 5, Geophysics, 43, 43-72, 1965.

Tsyganenko, N. A.: A model of the near magnetosphere with a dawn-dusk asymmetry 1. Mathematical Structure, J. Geophys.Res., 107(A8), 1179, doi:10.1029/2001JA000219, 2002a.

Tsyganenko, N. A.: A model of the near magnetosphere with a dawn-dusk asymmetry 2. Parameterization and fitting to observations, J. Geophys. Res., 107(A8), 1176, doi:10.1029/2001JA000220, 2002b.

Volwerk, M., Zhang, T. L., Nakamura, R., Runov, A., Baumjohann, W., Glassmeier, K.-H., Takada, T., Eichelberger, H. U., Carr, C. M., Balogh, A., Klecker, B., and Rme, H.: Plasma flow channels with ULF waves observed by Cluster and Double Star, Ann. Geophys., 23, 2929-2935, 2005,

http://www.ann-geophys.net/23/2929/2005/.

Walker, A. D. M., Ruohoniemi, J. M., Baker, K. B., and Greenwald, R. A.: Spatial and temporal behavior of ULF pulsations observed by the Goose Bay HF radar, J. Geophys. Res., 97, 12 187-12 202, 1992.
Waters, C. L., Takahashi, K., Lee, D.-H., and Anderson, B. J.: Detection of ultralow-frequency cavity modes using spacecraft data, J. Geophys. Res., 107(A10), 1284, doi:10.1029/2001JA000224, 2002.

Wright, A. N.: Dispersion and wave coupling in inhomogeneous MHD waveguides, J. Geophys. Res., 99(A1), 159-168, 1994.

Wright, A. N. and Rickard, G. J.: ULF pulsations driven by magnetopause motions: Azimuthal phase characteristics, J. Geophys. Res., 100, 23 703-23 710, 1995.

Wright, A. N. and Mann, I. R.: Global MHD Eigenmodes of the Outer Magnetosphere, Magnetospheric ULF Waves: Synthesis and New Directions, AGU Monograph Series, 169, 2006.

Zheng, Y., Lui, A. T. Y., Mann, I. R., Takahashi, K., Watermann, J., Chen, S.-H., Rae, I. J., Mukai, T., Russell, C. T., Balogh, A., Pfaff, R . F., and Rème, H.: Coordinated observation of field line resonance in the mid-tail, Ann. Geophys., 24, 707-723, 2006, http://www.ann-geophys.net/24/707/2006/. 